\title{
La polémique autour de bonjour/hi sur le web : vers la déconstruction du discours d'autorité
}

The online polemic surrounding bonjour/hi: towards the deconstruction of the discourse of authority

Chiara Molinari and Geneviève Bernard Barbeau

\section{(2) OpenEdition}

Electronic version

URL: http://journals.openedition.org/aad/5418

DOI: $10.4000 /$ aad. 5418

ISSN: 1565-8961

Publisher

Université de Tel-Aviv

\section{Electronic reference}

Chiara Molinari and Geneviève Bernard Barbeau, "La polémique autour de bonjour/hi sur le web : vers la déconstruction du discours d'autorité", Argumentation et Analyse du Discours [Online], 26 | 2021 , Online since 14 April 2021, connection on 16 April 2021. URL: http://journals.openedition.org/aad/ 5418 ; DOI: https://doi.org/10.4000/aad.5418

This text was automatically generated on 16 April 2021.

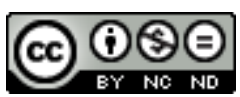

Argumentation \& analyse du discours est mis à disposition selon les termes de la licence Creative Commons Attribution - Pas d'Utilisation Commerciale - Pas de Modification 4.0 International. 


\title{
La polémique autour de bonjour/hi sur le web : vers la déconstruction du discours d'autorité
}

The online polemic surrounding bonjour/hi: towards the deconstruction of the discourse of authority

\author{
Chiara Molinari and Geneviève Bernard Barbeau
}

\section{Introduction}

1 Province francophone dans un Canada majoritairement anglophone, le Québec lutte depuis toujours pour assurer la place du français sur son territoire. L'anglicisation et le rapport de force inégal entre francophones et anglophones, qui ont caractérisé le paysage linguistique québécois jusqu'aux années 1960, se sont heurtés au barrage posé par l'aménagement linguistique mis en œuvre au Québec et qui a abouti, en 1977, à la promulgation de la Charte de la langue française (loi 101). Celle-ci non seulement attribue au français le rôle d'unique langue officielle du Québec, mais met au point des stratégies visant à sa diffusion, notamment dans le monde du travail et des affaires, dans l'affichage public et dans le système scolaire (Corbeil 2007).

2 Le paysage linguistique québécois n'est pas uniforme pour autant et le taux de bilinguisme français-anglais est en constante augmentation: le dernier recensement (2016) signale une augmentation de 7,7\% par rapport à 2011'. Par ailleurs, la proportion de Québécois ayant déclaré avoir le français comme langue maternelle a diminué au cours de cette période, bien que l'usage du français à la maison - comme langue unique ou en contexte plurilingue - soit demeuré stable. En revanche, la proportion de Québécois ayant déclaré l'anglais comme langue maternelle est en légère augmentation ${ }^{2}$. La présence de l'anglais est particulièrement évidente à Montréal, où le contact entre les groupes linguistiques est le plus fréquent et les pratiques linguistiques, les plus diversifiées. De ce fait, les discussions sur le rapport entre les langues et sur l'avenir linguistique du Québec portent plus souvent qu'autrement sur la 
situation montréalaise (Lamarre 2018). À cela s'ajoute la place centrale que joue Montréal à l'échelle du Québec en tant que métropole économique et culturelle, ce qui influence forcément la dynamique sociolinguistique québécoise (Blondeau et Remysen 2016). En 2016, le taux de bilinguisme français-anglais dans la métropole correspond à environ $60 \%$ et le tiers de la population emploie majoritairement l'anglais comme langue de travail ${ }^{4}$. En conséquence, malgré les tentatives menées sur le plan législatif pour favoriser le français comme langue commune d'usage public, l'anglais occupe toujours un rôle important, notamment dans la vie économique, ce qui ravive des tensions jamais surmontées qui resurgissent de façon récurrente dans l'espace public québécois.

\section{La formule bonjour/hi : point de départ de deux polémiques}

3 C'est ce qui s'est produit en novembre 2017, alors que le débat concernant l'équilibre délicat entre les deux langues a repris toute son ampleur lors de l'éclosion de ce que l'on peut qualifier d'«affaire bonjour/hi », qui fait référence à un rituel de salutation bilingue fréquemment employé dans les commerces montréalais pour accueillir les clients. L'affaire a comme origine une déclaration médiatique de Marie Montpetit, alors ministre de la Culture et des Communications et ministre responsable de la Protection et de la Promotion de la langue française, qui a qualifié cette formule de salutation «d'irritante pour les francophones ». Jean-François Lisée, alors chef du Parti québécois, parti de l'opposition officielle, en a profité pour demander à son homologue du Parti libéral, Philippe Couillard, alors premier ministre du Québec, ce qu'il pensait de cette expression qui, selon lui, représente le déclin du français à Montréal. Est dès lors convoquée l'idée, très souvent mobilisée dans les discussions sur la langue au Canada, selon laquelle le bilinguisme constitue une étape vers une inévitable assimilation linguistique (voir notamment Boudreau 2016). C'est à la suite de cet échange qu'un débat à ce sujet a éclaté à l'Assemblée nationale du Québec. Il faut préciser que le contexte sociolinguistique québécois était déjà tendu, à l'automne 2017, à la suite de nombreux épisodes où la question de la langue a été particulièrement mobilisée et qui ont été à l'avant-plan médiatique pendant plusieurs semaines. Dans ce contexte, l'affaire bonjour/hi constitue un prétexte pour nombre de politiciens, mais aussi de journalistes et de citoyens, pour revenir sur la question linguistique, qui déchire le Québec depuis longtemps et qui a été particulièrement présente dans l'espace public dans les semaines précédant le débat. C'est ainsi que le 30 novembre 2017, après des discussions parfois virulentes non seulement sur l'expression même, mais aussi - et surtout-sur la situation linguistique québécoise en général et montréalaise en particulier, les députés québécois, tous partis confondus, adoptent une motion suggérant que bonjour soit la formule de salutation privilégiée dans les commerces. Certes, une motion n'est pas une loi et n'a aucun effet légalement contraignant. Néanmoins, l'autorité que représente l'Assemblée nationale est suffisante pour que la nouvelle se répande, que les médias s'en saisissent et que l'on assiste à une véritable circulation des discours autour de la nouvelle, qui aboutit à son tour à une polémique, soit « un débat autour d'une question d'actualité, d'intérêt public, qui comporte des enjeux de société plus ou moins importants dans une culture donnée " (Amossy 2014 : 51), au fur et à mesure qu'on en a dévoilé les enjeux: linguistiques, politiques et 
économiques. Cette polémique a déjà fait l'objet d'une réflexion (Bernard Barbeau et Molinari 2021) où il a été question de déterminer l'appartenance de l'expression à la catégorie discursive des formules (Krieg-Planque 2009) et d'en dévoiler en même temps les enjeux socioculturels et idéologiques.

Or, le débat était loin d'être terminé. Au mois d'octobre 2019, deux ans après le déclenchement de la polémique et un an après l'arrivée au pouvoir d'un nouveau parti politique (Coalition Avenir Québec), le ministre responsable de la langue française, Simon Jolin-Barrette, connu pour ses positions conservatrices, est revenu sur la motion votée par l'Assemblée nationale en 2017 et a déclaré avoir l'intention d'aller plus loin et d'interdire bonjour/hi dans les commerces et dans les services publics de Montréal au profit du rituel français de salutation bonjour. Les mesures envisagées par le ministre pour mettre en œuvre son projet ne sont toutefois pas précisées : Jolin-Barrette évoque les résultats positifs obtenus par l'office québécois de la langue française ${ }^{5}$ avec les entreprises, mais n'annonce aucun plan concret. Cependant, de telles affirmations ne pouvaient passer inaperçues et le ministre ne pouvait espérer un accueil neutre. L'annonce de Jolin-Barrette a retenti dans les médias, où elle a suscité de fortes réactions à tel point qu'une nouvelle polémique s'est déclenchée. C'est à cette deuxième polémique que nous nous intéressons dans cet article, mais en privilégiant une autre perspective. Il ne sera plus question d'observer la manière dont la polémique s'élabore, mais plutôt d'interroger la façon dont se construit le discours de Jolin-Barrette - qui tente de s'imposer comme discours d'autorité - et les réactions qu'il suscite, tant au sein de l'institution politique que dans les médias et dans la population qui s'exprime dans la presse en ligne et sur les réseaux sociaux.

\section{Problématique et cadre analytique}

5 À partir de la banque de données Eureka et des mots-clés bonjour+hi, nous avons recensé, pour la période allant du $1^{\mathrm{er}}$ octobre 2019 au 31 janvier 2020, 200 articles de journaux francophones et anglophones publiés surtout au Québec, mais également ailleurs au Canada, ainsi que 1295 commentaires de lecteurs publiés à leur suite. Nous avons également interrogé les médias sociaux, ce qui nous a permis de relever les fils de discussion sur la page Facebook des journaux consultés où il était question de la formule, pour un total de 24000 commentaires, en plus de 922 gazouillis sur Twitter indexés sous le mot-dièse \#BonjourHi, de 137 commentaires sur Instagram et de 89 commentaires sur Youtube publiés à la suite de dix vidéos. Le nombre de réactions suscitées témoigne de l'ampleur de la polémique dans les médias, tant traditionnels que socio-numériques, et de l'intérêt qu'elle suscite. Pour des raisons de faisabilité, nous nous concentrons ici, après avoir présenté brièvement les réactions du monde politique et médiatique aux propos de Jolin-Barrette, sur les commentaires de lecteurs publiés sur le site web des journaux, sur Facebook et sur Twitter.

6 À partir de ce corpus hétérogène, qui se déploie sur plusieurs dispositifs ou plateformes et qui donne à voir la matérialité discursive par laquelle la seconde polémique autour de bonjour/hi prend forme, notre objectif est d'examiner la façon dont les discours sont construits par les sujets ayant une autorité légitime et, par la suite, appréhendés par les différentes communautés discursives qui se profilent dans les dispositifs en ligne, étant donné que ces derniers prévoient la participation d'usagers diversifiés. Par ailleurs, s'il est vrai que les médias sociaux ne permettent pas toujours de remonter à l'identité 
authentique des intervenants qui se cachent derrière des pseudonymes, l'histoire linguistique même du Québec nous légitime à poser l'existence de "groupement(s) social(-aux) légitimé(s)» (Douglas 1999), à savoir des regroupements culturels et sociaux dont la parole et les positionnements sont autorisés du fait de leur compétence et de leur expérience en tant que communauté inscrite dans un cadre historique particulier.

7 Notre hypothèse consiste à poser que les opinions individuelles permettent de dégager des dynamiques plus larges et qu'elles contribuent, de ce fait, à l'organisation d'un (ou de plusieurs) discours collectif(s). Ceux-ci seront orientés soit vers la construction d'un consensus autour du discours émis par les instances institutionnelles, soit vers l'élaboration d'un contre-discours antagoniste qui vise à le disqualifier, voire à le délégitimer pour proposer des représentations du monde différentes (Carbou 2015). Mais s'il est facile de poser que les participants au débat vont tantôt adhérer au discours institutionnel, tantôt s'y soustraire et le réfuter, il sera intéressant d'étudier les instances énonciatives ainsi que les modalités discursives à travers lesquelles ils expriment leurs considérations et se positionnent face à un discours d'autorité. La prise en compte de dispositifs différents (discours journalistiques et réactions des lecteurs dans la presse en ligne ainsi que dans les réseaux sociaux) permettra, en outre, de vérifier si les stratégies discursives d'acceptation ou de réfutation de l'autorité mises en œuvre sont les mêmes.

\section{Analyse}

\subsection{Le discours de Simon Jolin-Barrette : une autorité qui peine à s'affirmer}

8 Sur le plan discursif, nous sommes confrontées à un discours qui émane d'une autorité (un ministre) légitimée à le prononcer (Bourdieu 1982). Cependant, bien que le corpus recueilli ait des proportions importantes, il ne contient pas le discours de Jolin-Barrette dans son intégralité. L'accès à ses mots se fait par des extraits rapportés par les journalistes dans différents articles et dont la plupart se limitent à évoquer l'intention $\mathrm{du}$ ministre de légiférer afin d'interdire la formule bonjour/hi ${ }^{6}$. Cette occultation constitue déjà, à notre sens, un premier indice de la délégitimation dont les mots de Jolin-Barrette font l'objet. La volonté du ministre d'intervenir de façon concrète n'est rapportée qu'une fois :

À la question: comment comptez-vous vous y prendre afin de mettre à l'index l'expression «Bonjour-Hi ! »?, M. Jolin-Barrette a répondu : «Laissez-moi voir les possibilités.» "Ces défis [liés à l'effritement du français], je veux les relever [...] avec efficacité, pragmatisme et surtout afin que ce soit applicable. Et ça, c'est extrêmement important ", avait-il insisté quelques minutes plus tôt en Chambre (Marco Bélair-Cirino, "Simon Jolin-Barrette veut mettre à l'index le "Bonjourhi !" ", Le Devoir, 5 octobre 2019).

Néanmoins, Jolin-Barrette semble, lui aussi, incertain quant au pouvoir qui devrait lui être conféré par sa fonction. En effet, bien que son rôle de ministre responsable de la langue française devrait suffire à attribuer à ses mots l'autorité dont ils ont besoin pour devenir performatifs, Jolin-Barrette s'appuie à son tour sur des arguments émanant d'institutions difficiles à contester, que ce soit l'Assemblée nationale ou l'office québécois de la langue française : 
S'il est d'avis que les lois doivent être facilement "applicables ", le ministre JolinBarrette a dit vouloir tout de même donner suite aux motions adoptées unanimement à l'Assemblée nationale. La plus récente de ces motions, présentée par le Parti québécois, invitait « tous les commerçants et tous les salariés qui sont en contact avec la clientèle locale et internationale à l'accueillir chaleureusement avec le mot "bonjour" ». Il a cité des données de l'office québécois de la langue française (OQLF), selon lesquelles l'accueil en français à Montréal serait passé de $84 \%$ à $75 \%$ entre 2010 et 2017 (Janie Gosselin, «Québec aimerait interdire le "bonjour-hi" ", La Presse, 5 octobre 2019).

10 La convocation d'autres instances, notamment en matière de langue, ne suffit pas à assurer au discours du ministre l'autorité nécessaire pour s'imposer. En effet, les considérations de Jolin-Barrette ne reçoivent pas l'accueil prévu tant et si bien qu'il est aussitôt obligé de s'auto-corriger :

Lors d'une mêlée de presse, vendredi dernier, le ministre responsable de la langue française, Simon Jolin-Barrette, n'avait pas fermé la porte à légiférer pour interdire aux commerçants privés l'utilisation du «bonjour-hi ». Lors d'une conférence de presse dans sa circonscription, lundi, M. Jolin-Barrette a précisé qu'il souhaitait plutôt mettre en place des "incitatifs " pour que les commerces utilisent le mot «bonjour» seulement. "Le gouvernement du Québec doit prendre toutes les mesures, tous les incitatifs pour faire en sorte que les personnes soient accueillies en français dans les différents commerces, mais on ne légifèrera pas sur la question unique du "bonjour-hi" ", a-t-il dit (Hugo Pilon-Larose, "Québec ne légifèrera pas sur le "bonjour-hi" ", La Presse, 7 octobre 2019).

11 La coexistence de propos contradictoires contribue dès lors à une fragilisation du pouvoir du ministre.

12 Face à ce recul, signalé par les journalistes et par des députés et militants du Parti québécois qui réclament des interventions concrètes au profit de la langue française, le ministre propose un discours qui exploite le procédé métaphorique pour augmenter sa force de persuasion et s'auto-attribuer une allure autoritaire, tout en restant mystérieux :

Au député Arseneau, qui lui a lancé en Chambre : «À quand les actions ? [...] On n'a rien à se mettre sous la dent !», M. Jolin-Barrette a répondu : «Le dîner n'est pas encore servi, mais il sera servi très bientôt et il y aura de la nourriture pour en manger énormément ». La veille, $\mathrm{M}$. Arseneau avait relevé le recul du ministre sur l'enjeu du « Bonjour-hi ». Simon Jolin-Barrette avait dû corriger le tir, après avoir suggéré une interdiction du «Bonjour-hi » dans les commerces pour faire en sorte que le français soit la seule langue d'accueil (Caroline Plante, «Protection de la langue française: Jolin-Barrette promet des mesures costaudes ", L'Actualité, 8 octobre 2019).

13 L'énoncé métaphorique de Jolin-Barrette correspond à une promesse (Angenot 2013 : 76), dont l'énonciateur se sert pour essayer de rétablir son autorité, menacée par le recul précédent. Cependant, non seulement celle-ci n'est pas pleinement assumée de la part de l'énonciateur, qui préfère la modalité de l'effacement énonciatif à un engagement direct et personnel, mais ne se traduira en aucune action concrète.

En somme, si Jolin-Barrette tente de construire un discours d'autorité, ce dernier n'est pas pleinement assumé et n'aboutit pas à un résultat tangible. Cet échec de la construction $\mathrm{du}$ discours d'autorité $\mathrm{du}$ ministre n'est toutefois peut-être pas complètement étranger à la façon dont les journalistes ont rapporté ses mots ni, on le verra, aux réactions suscitées par son annonce. 


\subsection{Les réactions institutionnelles : de l'interdiction au silence} brièvement sur celles que l'on pourrait qualifier d'institutionnelles en ce qu'elles émanent d'énonciateurs jouant un rôle officiel dans l'arène politique. Bien qu'elles ne soient pas nombreuses, ces réactions attirent tout de même l'attention des médias et nous paraissent intéressantes à souligner. En général, elles concernent le premier ministre québécois, François Legault, et le premier ministre canadien, Justin Trudeau. Toutes deux se déploient dans le même sens et témoignent d'une prise de distance par rapport aux déclarations de Jolin-Barrette. François Legault intervient de façon directe pour nier les affirmations du ministre et affirmer que son gouvernement ne légifèrerait pas en la matière, déconstruisant de ce fait l'autorité du ministre. En revanche, Justin Trudeau s'exprime selon des modalités différentes, notamment par une prise de position silencieuse mais non moins performative, refusant de se prononcer sur l'affirmation de Jolin-Barrette. Sur le plan discursif, le silence de Trudeau est loin de traduire une attitude neutre (Barbet et Honoré 2013). Si le silence «n'est pas une substance mais une relation" (Le Breton $1997: 80$ ), dans ce cas spécifique, il relève plutôt d'une prise de distance, voire d'une indifférence délégitimante à l'égard du discours du ministre dont les affirmations sont désavouées. On constate que la disqualification du discours de Jolin-Barrette vient de la part de sujets ayant un rôle plus important : loin de l'appuyer, ceux-ci s'en éloignent en faisant ainsi chanceler son autorité. La seule réaction positive aux propos de Jolin-Barrette est celle de l'ancienne première ministre québécoise Pauline Marois: si elle croit qu'il n'est pas possible d'adopter une telle loi interdisant la formule bonjour/hi, elle reconnait la valeur symbolique et morale des actes du ministre pour la protection de la langue française.

\subsection{Les réactions de la communauté}

Les réactions de la communauté à l'égard des déclarations du ministre responsable de la langue française sont nombreuses et hétérogènes, et ce, tant du point de vue des dispositifs concernés que des énonciateurs. Par ailleurs, le retentissement dont le discours de Jolin-Barrette fait l'objet dans les médias - que ce soit la presse en ligne ou les médias sociaux - produit un effet de réification qui se fait gage d'authenticité de la nouvelle rapportée (Charaudeau 2006: 5). Dans ce cadre, nous distinguerons les attitudes des journalistes à l'intérieur de leurs textes de celles des lecteurs qui s'expriment dans les commentaires à la suite des articles et dans les réseaux sociaux.

\subsubsection{Les journalistes : une nouvelle autorité ?}

L'autorité du ministre n'est pas à l'abri non plus chez les journalistes : s'ils ne possèdent pas le pouvoir institutionnel, ces derniers sont tout de même responsables du pouvoir médiatique dont l'influence est bien connue (Charaudeau 2006) et sont loin d'appuyer les mots prononcés par Jolin-Barrette. Certains, il faut le reconnaître, se limitent à une narration neutre des événements. D'autres, en revanche, sèment le doute, voire ridiculisent les mots du ministre. Les stratégies discursives mobilisées à cette fin sont différentes. Parfois, les journalistes exploitent des bribes de discours rapporté par des instances énonciatives qui ne sont pas choisies au hasard. Ainsi, après avoir construit son article en alternant la chronique des événements avec le point de vue de différents

Argumentation et Analyse du Discours, 26 | 2021 
acteurs sociaux aux opinions opposées sur la question, la journaliste Janie Gosselin conclut par un paragraphe dont le titre, constitué d'un îlot textuel ayant une valeur axiologique négative importante (Kerbrat-Orecchioni 1980), jette un fort discrédit sur le discours de Jolin-Barrette :

« Ridicule »

Selon l'avocat Harold Staviss, interdire le «bonjour-hi» serait «vraiment ridicule ». « Montréal, tout le monde sait que c'est une ville bilingue et multilingue, a-t-il souligné. Pourquoi ne peut-on pas dire "bonjour-hi" ? C'est de la courtoisie pour les francophones, les anglophones et les allophones. " ("Québec aimerait interdire le "bonjour-hi" ", La Presse, 5 octobre 2019)

Ce discrédit du discours du ministre constitue, pour reprendre les termes de Laforest et Moïse (2013), un acte de condamnation du faire, c'est-à-dire qu'il sert à disqualifier un individu en raison de ses actions. Or, comme le souligne Vincent (2013 : 40), même quand ce sont des gestes ou des propos bien précis qui sont pointés du doigt, comme c'est le cas ici de la déclaration de Jolin-Barrette, « il n'en demeure pas moins que, par extension, c'est souvent la légitimité d'autrui, de son statut, de ses actions ou de ses croyances qui est contestée ». Ainsi, d'une condamnation du faire, on passe à une condamnation de l'être, où l'individu même est disqualifié. Par ailleurs, "ridicule", employé autant dans sa valeur d'adjectif que de substantif, revient à plusieurs reprises dans le discours journalistique - citons notamment "Le ridicule ne tue pas, mais... » (Paul Journet, La Presse, 8 octobre 2019) et "Interdire le "Bonjour-hi" ou le ridicule péremptoire » (Micheline Marier, Le Devoir, 7 octobre 2019) - pour décrire les propos de Jolin-Barrette (ou, par corollaire, le ministre même). Cela permet d'observer un phénomène de reprise et de circulation discursive qui contribue à élaborer une représentation négative du discours en question.

Les stratégies de l'ironie et du sarcasme sont elles aussi fréquemment mobilisées pour démonter, petit à petit, le discours du ministre. Par exemple, après avoir exploité le procédé de l'ironie interdiscursive (Bres 2010) en affirmant que légiférer pour interdire bonjour/hi est " une riche idée ", le chroniqueur Yves Boisvert («Projet de loi contre toutes les choses vraiment énervantes ", La Presse, 7 octobre 2019) soumet à son lectorat une énumération de propositions extravagantes qu'il suggère d'associer au projet de loi. Cette association, qui suscite inévitablement l'approbation et le sourire du lectorat sans pour cela avoir un effet purement ludique, contribue à faire ressortir l'absurdité de la proposition de Jolin-Barrette et à jeter le discrédit sur le ministre. Par conséquent et conformément aux considérations de Ducrot (1984), non seulement parler ironiquement correspond-il à énoncer quelque chose dont on n'assume pas la responsabilité, mais exercer l'ironie signifie également mettre en œuvre un dispositif énonciatif complexe auquel participent plusieurs acteurs. Grâce au procédé rhétorique de l'ironie, l'énonciateur parvient ainsi à établir une relation de connivence et de complicité avec les lecteurs contre une cible que ceux-ci sont censés connaître. On pourrait s'interroger sur les conséquences de cette relation : les lecteurs se sentent-ils autorisés davantage à réagir et à s'exprimer, que ce soit dans les réactions aux articles ou dans les réseaux sociaux ? Nous y reviendrons en 3.3.2.

Le seul exemple journalistique qui s'apparente de prime abord à un contre-discours visant véritablement à répondre à Jolin-Barrette est celui du chroniqueur Mathieu Bock-Côté ("La première gaffe de Jolin-Barrette», Le Journal de Montréal, 8 octobre 2019) qui, après avoir déclaré ne pas partager les projets du ministre (qualifiés de "croisade ridicule»), élabore un discours où il propose ce qu'il présente comme des 
pistes pour la défense du français au Québec. Or, celles-ci, toutes introduites au moyen de l'artifice rhétorique de la répétition du syntagme «il peut " ( Jolin-Barrette peut $\mathrm{X} »$ ), culminent plutôt vers un discours ouvertement anti-immigration, où le recul du français dans la sphère publique est imputé aux nouveaux arrivants, amalgame fréquent dans le discours public québécois. L'objectif est alors moins de répondre au ministre - d'ailleurs en faveur lui aussi d'un important resserrement de la politique d'immigration - que de présenter un argumentaire hostile et discriminatoire qui s'inscrit dans une idéologie conservatrice pour laquelle le chroniqueur est bien connu (Fortier 2019).

\subsubsection{L'autorité des communautés 2.0}

21 Les affirmations de Jolin-Barrette ont suscité un fort écho dans la population, particulièrement dans les médias sociaux, qui favorisent la prise de parole d'un nombre croissant d'individus dont on ne connaît pas forcément l'identité, selon l'une des règles à la base des réseaux sociaux où les sujets peuvent s'exprimer sous le masque d'un pseudonyme. La prolifération des nouvelles technologies a abouti à deux phénomènes liés par une relation de cause à effet : si, d'une part, l'accès au web a accéléré de façon importante le rebondissement et la "disponibilité sociale d'une grande variété de discours sur une grande variété de thèmes " (Carbou 2015, en ligne), elle a aussi favorisé, de l'autre, l'accès à ces nouvelles de la part d'un public qui devient, à son tour, responsable de cette circulation et auquel les nouveaux médias garantissent aussi la possibilité d'intervenir et de devenir un sujet actif dans la fabrication de contenus. Or, notre intérêt initial était d'examiner la façon dont le public d'internautes se positionne par rapport aux paroles du ministre. Celles-ci sont-elles considérées comme un discours émanant d'une autorité légitime ou bien les internautes s'emparent-ils plutôt du discours du ministre afin de construire un contre-discours d'autorité ? Par quels instruments? Et qui leur octroie ce pouvoir?

L'étude du corpus a permis de relever un double positionnement: les internautes expriment certes leur avis par rapport aux considérations du ministre, mais répondent aussi souvent aux questions concernant les déclarations du ministre soulevées par les journalistes. Précisons que cette attitude concerne aussi bien les commentaires que les lecteurs publient à la suite des articles en ligne que leurs réactions dans les réseaux sociaux. Là aussi, en effet, les interventions ne jaillissent pas spontanément, mais agissent généralement en tant que réponse à un article diffusé en ligne.

Les commentaires des lecteurs sont extrêmement hétérogènes, mais reproduisent grosso modo les catégories relevées plus haut, témoignant d'une circulation entre discours journalistiques et réactions citoyennes. Rares sont les internautes qui appuient le discours du ministre. Plus fréquents sont les cas où ils critiquent ses propos considérés comme superficiels et non indispensables, voire le ministre lui-même, considéré comme incompétent : 
Benoît @ IY'ont rien de plus important à faire? Niaisage. Mi piace $\cdot$ Rispondi $\cdot 43 \mathrm{~s}$

- André : Ne vous inquiétez pas ils font des choses importantes et au détriment de la population. Ils se couvrent avec ces conneries populistes.

Mi piace $\cdot$ Rispondi $\cdot 43 \mathrm{~s}$

(6) Françoise : 3 C'est symbolique mais important.

Mi piace - Rispondi $\cdot 43 \mathrm{~s}$

Benoît C. Quand tu es rendu à gérer les salutations dans un commerce, ce n'est pas important, c'est ridicule, petit et de mauvais aloi pour l'avenir et le vivre-ensemble. Et côté symbolique, ils représentent assez bien une symbolique colon, raciste et rétrograde.

Mi piace $\cdot$ Rispondi $\cdot 43 \mathrm{~s}$

(https://www.facebook.com/ledevoir/posts/10157207736065528)

L'extrait rapporté ci-dessus rappelle le schéma déjà présenté lors de l'analyse des discours des journalistes : la décision du ministre est dénigrée et qualifiée de « connerie populiste " ou encore de "ridicule", adjectif dont le retour confirme la circulation discursive. Notons d'ailleurs que ce sont ces réactions négatives qui, dans cet extrait, reçoivent le plus de marques d'approbation sous forme d'émoticônes. Dans cet échange, l'unique réaction en faveur de Jolin-Barrette ("C'est symbolique mais important») reçoit des réactions moqueuses qui témoignent à leur tour de la dévalorisation des affirmations du ministre. Celle-ci dépasse parfois la dimension purement discursive pour s'attacher au personnage qui est non seulement ridiculisé («le petit Barrette », relevé dans ce même fil de commentaires Facebook) mais qui devient la cible d'insultes et de qualifications péjoratives (Laforest et Vincent 2004) :

\section{Johanne L.... Petit dictateur. OK, BYE !}

Mi piace $\cdot$ Rispondi $\cdot 43 \mathrm{~s} \cdot$ Modificato

\section{Reff : i Oh l'idiot ce ministre pathétique \\ Mi piace $\cdot$ Rispondi $43 \mathrm{~s}$}

Mais ce qui nous paraît plus intéressant à explorer, ce sont les contre-discours élaborés par les internautes. Ces derniers ne se limitent pas à condamner le ministre, mais proposent souvent des arguments qui s'appuient, dans la plupart des cas, sur des idées 
reçues circulant depuis longtemps et plongeant leurs racines dans un discours socioculturel ancien. Nombreux sont aussi ceux qui ne se limitent pas à la critique mais se servent $\mathrm{du}$ web pour avancer leur proposition. Plusieurs pistes se dégagent et s'éloignent parfois du discours initial : celui-ci est souvent dépassé par, voire inclus dans, un discours plus large concernant les relations conflictuelles entre français et anglais au Québec et, de façon plus générale, au Canada. Considérons à ce propos les réactions à la chronique de Sophie Durocher dans Le Journal de Montréal. Pour la chroniqueuse, les affirmations de Jolin-Barrette ne sont qu'un prétexte pour entamer une réflexion au sujet des solutions pour défendre la langue française. Et c'est dans cette voie que s'engagent les internautes dont les interventions ne mentionnent même plus les mots du ministre, dont la ridiculisation - et la délégitimation qui s'ensuit - sont assurées en premier lieu par la chroniqueuse elle-même :

Sugar Sammy a tweeté vendredi, au sujet de la controverse Bonjour-Hi : «Mon nouveau show québécois s'écrit tout seul! ».

C'est sûr que pour Sugar Sammy, l'équipe du Bye Bye, et la plupart des humoristes du Québec, une loi anti-Hi, c'est du bonbon. Ils sont déjà en train d'écrire leurs blagues sur une Police de la langue qui collerait des contraventions au moindre salut bilingue. Mais, blague à part les amis, on fait quoi pour régler ce problème-là ? Avez-vous une solution? («Bonjour-hi, ce n'est pas (juste) une blague », Le Journal de Montréal, 7 octobre 2019)

Dans ce cas spécifique, la légitimation du contre-discours proposé par les internautes est possible grâce à la chroniqueuse (et donc grâce aux médias) : alors que le ministre est ridiculisé, Sophie Durocher s'adresse directement à son lectorat, qui est appelé à proposer des réponses. Celles-ci s'inscrivent dans l'arrière-plan socioculturel et identitaire québécois: en d'autres termes, les intervenants, souvent à travers une modalité dialogale, réactualisent quelques-unes des idées à la base du débat linguistique québécois, ce qui n'évite pas des échanges polémiques marqués par une dichotomisation des arguments et une polarisation des groupes qui s'affrontent. Ces représentations sont évoquées par le biais d'un discours où la dépersonnalisation énonciative (Rabatel 2004) - obtenue par l'emploi des formes infinitives et du présentatif «c'est» - alterne avec des manifestations timides de l'énonciateur. Pour certains, il faut améliorer la qualité du français :

\section{Marc Di}

Je me pose la même question que vous Mme Durocher: quel est le remède? I| faut certainement recommencer à valoriser la beauté de la langue française. Je pense à ceux qui ont cette manie de croire qu'li faut absolument utiliser des mots anglais pour avoir un titre accrocheur, un nom tape-à-l'oeil ou simplement sonner plus «hot». À mon avis c'est complètement faux, il y a assurément toujours une façon de faire joli et distingué avec des mots français. Bien maîtriser le français, c'est se donner un avantage afin de mieux se démarquer des autres.

Pour d'autres, en revanche, la formule bonjour/hi constitue une marque de respect à l'égard des anglophones :

\section{Marc Cl.}

Sincèrement le bonjour/hi est respectueux envers notre touriste de langue anglaise. La personne anglaise étrangère se sent plus alèse avec le bonjour/hi qu'un simple bonjour "La laissée se sentir chez eux chez nous". Le commerçant ne travaillant pas pour la langue, travaille pour être prospère dans les affaires de son établissement. Le bonjour/hi n'est pas contre le français ni pour l'anglais, il est pour le rendement "Profil" des établissements commerciales. On n'a pas à gripper dans les rideaux pour ça. 
D'autres arguments sont étayés au fur et à mesure dans les interventions : un article publié sur la page Facebook du journal Le Devoir concernant le projet de Jolin-Barrette est suivi de nombreux commentaires donnant lieu à des échanges virulents. Toutefois, contrairement à nos attentes, les internautes ne parviennent, dans ce cas spécifique, à construire aucun contre-discours, mais se limitent à prendre position pour ou contre la formule bonjour/hi. Face à l'affrontement de ces discours dichotomiques qui exploitent tantôt la violence verbale (Moïse et al. 2008) tantôt une ironie délégitimante, doit-on poser que les techno-discours (Paveau 2015) qui se développent dans les réseaux sociaux servent à minimiser la portée de l'autorité légitime sans pour autant lui opposer une alternative?

En fait, si les réseaux sociaux sont un espace où la polémique se déploie facilement, les contre-discours sont tout de même présents et ne s'élaborent pas seulement par le biais d'une neutralité discursive (apparente). Sur la page Facebook de la Presse, les internautes réagissent à un article concernant l'interdiction du bonjour/hi: la plupart des interventions exploitent la modalité de l'ironie et du sarcasme en jouant avec les possibilités offertes par le techno-discours (émoticônes, images, mèmes). C'est le cas de cette intervention, où l'internaute se moque de l'interdiction du bonjour/hi en évoquant la présence d'anglicismes dans la variété québécoise du français, ce qui suscite le rire, manifesté par les émoticônes, des lecteurs :

\section{Watthieu :.......... Jveux pas qu'on parle anglais mais} j'ai des NEWS pour toi.

\section{J'aime · Répondre $\cdot 35 \mathrm{sem}$}

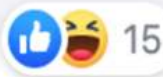

(https://www.facebook.com/LaPresseFB/posts/10159361566688312)

Il n'en reste pas moins que, souvent, le discours concernant la qualité de la langue française - qui, dans le premier commentaire ci-dessous, joue le rôle de contre-discours opposé au projet du ministre - est aussi mobilisé au cours d'échanges où les sujets se mettent en jeu, que ce soit sur un plan personnel ou communautaire :

\section{Mustapha :- $\quad$ Pour protéger ta langue comme tu dis, il faut savoir d'abord la parler correctement. Et sur ce plan, ton premier ministre lui-même est en net déficit.}

(https://www.facebook.com/LaPresseFB/posts/10159361566688312)

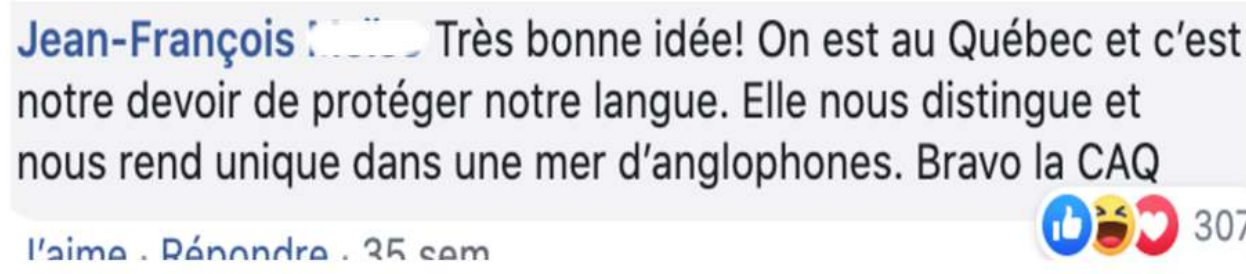

(https://www.facebook.com/LaPresseFB/posts/10159361566688312)

31 L'emploi du «nous" inclusif, dans le deuxième commentaire, contribue à rappeler l'existence d'un ethos collectif (Amossy 2010) qui s'appuie sur la valeur identitaire de la langue. 
32 En ce qui a trait aux tweets contenant le mot-dièse \#BonjourHi, ils sont extrêmement nombreux et ne s'arrêtent pas à l'automne 2019. Depuis les déclarations de JolinBarrette, le mot-dièse est repris de façon régulière et dépasse la période du débat tant et si bien qu'il revient encore aujourd'hui, ce qui contribue à renforcer l'hypothèse selon laquelle un événement discursif s'est peu à peu élaboré autour de la formule. Le mot-dièse remplit, à ce titre, un rôle fondamental, en ce qu'il réunit une communauté virtuelle, changeante et provisoire, autour du même sujet (Mercier 2015 : 149). La nature bilingue des tweets - des tweets en français alternent avec des tweets en anglais, et cette alternance se produit souvent tout au long des réponses à un même message initial - s'inscrit dans cette perspective : francophones et anglophones (ou, du moins, usagers s'exprimant dans ces deux langues) se réunissent autour du même sujet.

Les études au sujet des tweets et de la twittosphère soutiennent, en général, que ce dispositif «se prête très bien à un usage polémique, parce qu'il est à la fois une technologie de l'affirmation de soi et de mobilisation sociale » (Mercier 2015 : 146). Or, s'il est vrai que les réseaux sociaux conviennent particulièrement à l'expression de soi, il n'en reste pas moins que les tweets contenant le mot-dièse \#BonjourHi, ou encore la formule sans le mot-dièse, et réunis dans la période qui va de début octobre 2019 jusqu'à fin janvier 2020 ne constituent pas forcément un espace où se déploient les échanges polémiques. Les positionnements en faveur ou en défaveur de la formule alternent, mais n'aboutissent que rarement à de véritables confrontations, sans pour autant que les participants ne parviennent à un accord. Twitter, dans le cas qui nous concerne, apparaît plutôt comme un espace favorable à l'expression de soi et de ses opinions, et moins comme une remise en cause du discours d'autorité, du moins dans les premiers temps de la polémique.

L'ironie et le sarcasme, renforcés par le recours aux émoticônes, traversent la twittosphère et dépassent l'épisode concernant la volonté de légiférer contre le bonjour/hi annoncée par Jolin-Barrette. Ici, l'auteure du tweet évoque la Loi sur la laïcité de l'État, adoptée quelques mois auparavant par le gouvernement québécois et jugée discriminatoire envers les minorités religieuses. L'association entre cette loi et les propos du ministre concernant le bonjour/hi participe à la construction d'un discours qui, plus largement, vise à condamner l'ensemble de la société québécoise :

Diane C

@c

\section{Au Québec est ce que ont va envoyer en prison une enseignante voilé qui va osé dire \#BonjourHi, la haute trahison quoi 0 Calice que nous sommes dès incultes au Québec. Chanceuse d'avoir reçu mon éducation avant la réforme scolaire et je l'ai reçu en Anglais en plus \#GetLostBye \\ Traduci il Tweet}

7:56 PM $\cdot 5$ ott $2019 \cdot$ Twitter for Android 
télargissement est typique des échanges recueillis sur Twitter où, au-delà des annonces de Jolin-Barrette, des discussions parfois virulentes concernant la question linguistique et politique québécoise se donnent à voir et font état des tensions entre les individus et entre les groupes.

Il faudra attendre un an environ, jusqu'en novembre 2020, pour assister à une véritable remise en cause de l'autorité de Jolin-Barrette. En effet, dans la mesure où Twitter permet d'évaluer très rapidement la circulation discursive grâce aux mots-dièses, il a été possible de se pencher sur des tweets récents. Si ceux-ci dépassent la période établie au début de notre réflexion, il n'en est pas moins vrai que les derniers tweets parus suscitent deux réflexions. La première concerne l'appartenance du bonjour/hi à la catégorie des événements discursifs. En effet, les tweets au sujet des tentatives non réussies du ministre de renforcer le français en recul sur l'anglais se multiplient :

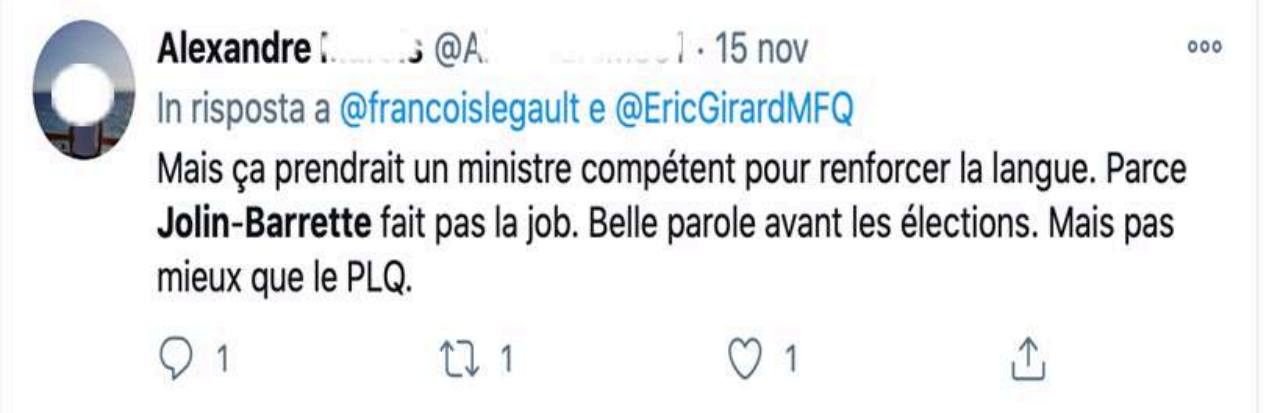

37 À la suite de la publication des résultats d'un sondage sur la situation du français à Montréal, l'automne 2020 a en effet été marqué par une série de discussions - tant dans l'espace médiatique que dans l'arène politique provinciale et fédérale - sur le recul du français dans la région métropolitaine. S'il n'a plus été question du rituel de salutation bilingue et des tentatives de Jolin-Barrette pour empêcher son usage un an auparavant, on remarque que les internautes réinvestissent l'espace numérique et s'en servent pour attaquer la crédibilité du ministre par rapport à sa gestion de l'ensemble du dossier linguistique dont il a la responsabilité. La deuxième réflexion concerne la nature fluide des processus de construction et de déconstruction de l'autorité dans les réseaux sociaux: la réactivation du discours au sujet de la défense du français liée à un événement social particulier permet de revenir sur des événements précédents et de renforcer, par conséquent, un discours déjà existant.

Sur un plan méthodologique, les internautes s'interrogent sur le rôle et sur l'autorité du ministre en s'appuyant souvent sur la citation d'extraits d'articles de la presse ou d'autres tweets. La polémique visant à le discréditer existe et se déploie à travers la circulation des discours sans pour autant qu'un dialogue ou un échange entre les internautes s'instaure : 
Claude. $\quad @ \quad \ldots \ldots+17$ nov

«Simon Jolin-Barrette, n'en finit plus de promettre des « mesures costaudes », qu'on attend depuis deux ans, mais qui doivent toujours s'effacer devant des priorités plus urgentes. Plus on attend, plus il sera difficile de renverser la vapeur » \#Français

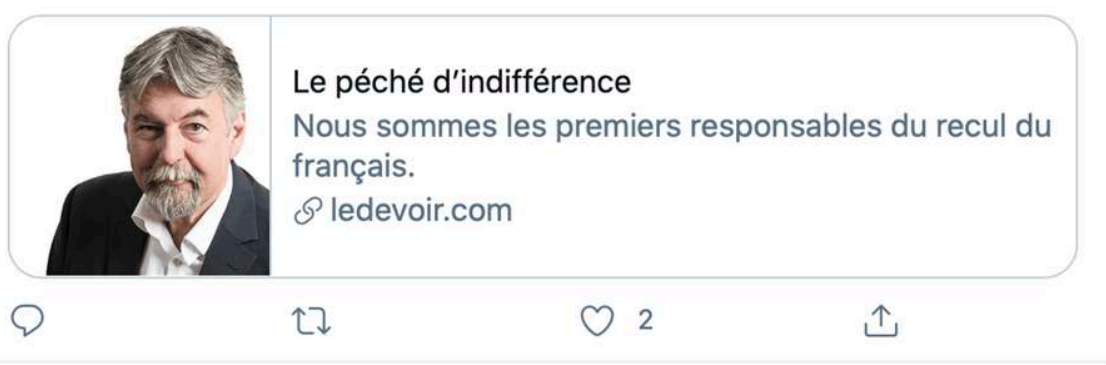

L'internaute cite ici un extrait de l'article du Devoir affiché sous son tweet, en anticipant et en mettant en valeur la partie saillante, à ses yeux, du texte partagé. L'aperçu à valeur cataphorique de l'article reproduit la modalité citative (Cagninelli 2020) et fonctionne comme contextualisation des dires rapportés qui décrédibilisent l'autorité de Jolin-Barrette et, par là, constitue une sorte de certification, de caution de ces dires. Le seul ajout du locuteur est représenté par le mot-dièse \#Français, qui peut servir tant à indexer le contenu du tweet qu'à remplir une fonction sociale d'affiliation ou d'identification à une communauté linguistique.

En revanche, le tweet ci-dessous s'inscrit, en quelque sorte, dans la modalité réactive : le texte de l'internaute associe en effet la citation de l'article de La Presse affiché à un commentaire ironique. En d'autres termes, ce segment textuel ne se limite pas à établir une redondance avec l'aperçu de l'article: l'internaute montre sa maîtrise du patrimoine littéraire de langue française (rappelant ce que Paveau (2006) qualifie " d'appel aux pères », mode d'appel sollicitant la mémoire discursive) et se place ainsi, implicitement, du côté des défenseurs de la langue, de ceux qui la sauvegardent et, en même temps, elle décrédibilise le ministre en condamnant son inaction : 
Drouin [ @ @ 1.14 nov $\quad 00$

Langue française : En attendant Simon Jolin-Barrette - Ou en attendant

Godot? lapresse.ca/debats/opinion... via @lp_lapresse

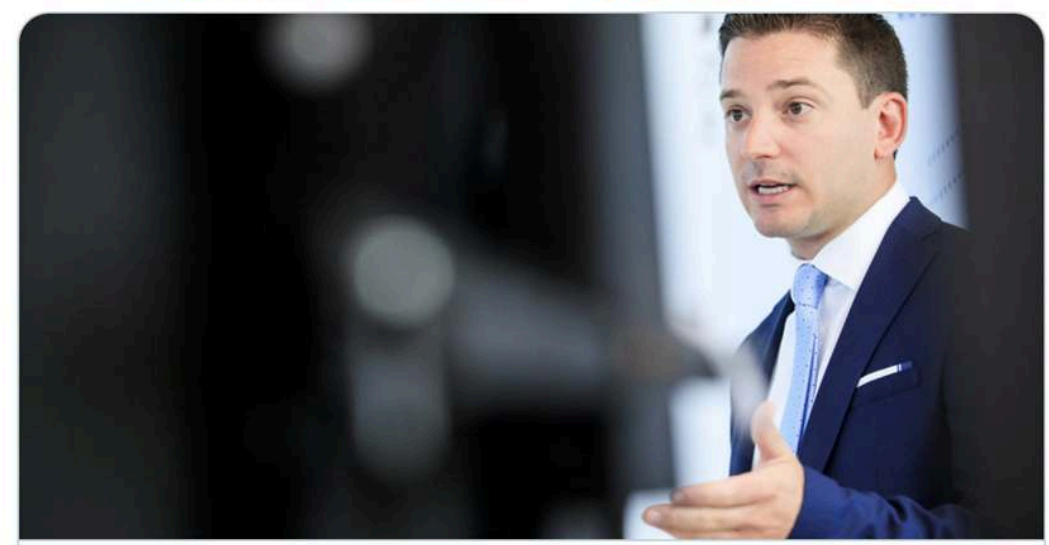

Langue française | En attendant Simon Jolin-Barrette

II y a deux ans et des poussières, l'arrivée au pouvoir de la troupe de François Legault a provoqué une véritable aération de la maison ...

$\mathcal{S}$ lapresse.ca

D $377 \quad$ ○ 7 个

41 Dans ces deux exemples, la déconstruction de l'autorité se fait donc par l'opposition à Jolin-Barrette de techno-discours véhiculant des valeurs partagées autour de la langue française. Le ton polémique est bien présent, mais résulte d'une circulation discursive que nous pourrions qualifier d' « augmentée », en ce que les mêmes textes et les mêmes citations reviennent à plusieurs reprises, et non pas d'échanges ayant une valeur argumentative particulièrement marquée ou contenant des traces de violence verbale.

\section{Conclusion}

42 L'autorité, qualifiée par Hannah Arendt (1972) de capacité d'obtenir l'obéissance « sans recourir à la contrainte par la force ou à la persuasion par arguments ", se voit de plus en plus disputée par d'autres moyens de légitimité (Le Deuff 2006 : 2), voire par d'autres instances plus ou moins légitimes.

L'analyse de la deuxième polémique autour de la formule bonjour/hi a permis de montrer d'une part les tentatives vaines de Simon Jolin-Barrette de construire son autorité et de l'autre les modalités discursives mobilisées pour, dans la majorité des cas, attaquer l'autorité du ministre. Ces modalités ne sont certes pas foncièrement différentes d'un dispositif médiatique à l'autre. En effet, que ce soit par le recours au silence, à la négation ou à l'ironie, par la condamnation des propos du ministre (et de là du ministre même) ou par l'élaboration de contre-discours, les participants au débat tant les journalistes que la population - contribuent à la délégitimation du ministre. Néanmoins, l'analyse révèle que ce sont les discours citoyens, dans les commentaires dans la presse comme dans les médias sociaux, qui permettent le mieux d'observer la construction de contre-discours, fondés pour la plupart sur des enjeux socioculturels dépassant le cadre initial du débat. Ainsi, alors que les journalistes et chroniqueurs démontent le discours de Jolin-Barrette et jettent le discrédit sur le ministre, la 
population confère une dimension polémique plus importante aux échanges en les inscrivant dans un arrière-plan sociohistorique chargé qui va bien au-delà des propos $\mathrm{du}$ ministre. Ajoutons à cela que les possibilités techno-discursives offertes par les médias sociaux contribuent à la force de frappe des discours qui y circulent. À ce sujet, notons que c'est par Twitter que l'on peut observer la création d'un véritable événement discursif autour du bonjour/hi, le mot-dièse servant alors autant à parler de la polémique que, un an plus tard, à attaquer la crédibilité de Jolin-Barrette. En somme, médias traditionnels et réseaux sociaux légitiment la prise de parole de chacun et favorisent, par conséquent, la remise en cause de la confiance sur laquelle s'appuyait normalement l'autorité institutionnelle et politique.

\section{BIBLIOGRAPHY}

Amossy, Ruth. 2010. La présentation de soi. Ethos et identité verbale (Paris : PUF)

Amossy, Ruth. 2014. Apologie de la polémique (Paris : PUF)

Angenot, Marc. 2013. Rhétorique de la confiance et de l'autorité (Montréal : Chaire James-McGill

d'étude du discours social, Discours social vol. 44)

Arendt, Hannah. 1972. La crise de la culture (Paris : Gallimard)

Barbet, Denise \& Jean-Paul Honoré. 2013. « Ce que se taire veut dire. Expressions et usages

politiques du silence ", Mots 103, 7-21

Bernard Barbeau, Geneviève \& Chiara Molinari. 2021. « Bonjour/hi, ou quand la polémique arrive par les mots ", Bernard Barbeau, Geneviève, Franz Meier \& Sabine Schwarze (éds). Conflits sur/ dans la langue : perspectives linguistiques, argumentatives et discursives (Francfort : P. Lang), 57-76

Blondeau, Hélène \& Wim Remysen. 2016. « Du local au global : pratiques et idéologies

linguistiques en contexte montréalais », Cahiers internationaux de sociolinguistique 10, 13-17

Boudreau, Annette. 2016. « Les idéologies linguistiques chez quelques chroniqueurs du journal Le Devoir de 1990 à 2015 : examen d'un discours d'autorité », Francophonies d'Amérique 42-43, 125-140 Bourdieu, Pierre. 1982. Ce que parler veut dire. L'économie des échanges linguistiques (Paris: Fayard)

Bres, Jacques. 2010. «L'ironie, un cocktail dialogique », Neveu, Frank Valelia Muni Toke, Jacques Durand, Lorenza Mondada \& Sophie Prévost (éds). Congrès Mondial de Linguistique Française (Paris : Institut de linguistique française), 695-709

Cagninelli, Claudia. 2020. « Le débat public sur Twitter : construction énonciative et discursive des tweets intégrant des liens URL », Repères-Dorif 22, http://dorif.it/ezine/

ezine_printarticle.php?id=484 (consulté le 14 mars 2021)

Carbou, Guillaume. 2015. « Des contre-discours aux contre-mondes : l'exemple des commentaires d'internautes autour de l'accident de Fukushima », Semen 39, https://journals.openedition.org/ semen/10478 (consulté le 14 mars 2021)

Charaudeau, Patrick, 2006. «Discours journalistique et positionnements énonciatifs. Frontières et dérives », Semen 22, https://journals.openedition.org/semen/2793 (consulté le 14 mars 2021) 
Corbeil, Jean-Claude. 2007. L'embarras des langues. Origine, conception et évolution de la politique linguistique québécoise (Montréal : Québec Amérique)

Douglas, Mary. 1999. Comment pensent les institutions (Paris : La Découverte)

Ducrot, Oswald.1984. Le dire et le dit (Paris : Minuit)

Fortier, Mark. 2019. Mélancolies identitaires. Une année à lire Mathieu Bock-Côté (Montréal : Lux)

Kerbrat-Orecchioni, Catherine. 1980. L'énonciation. De la subjectivité dans le langage (Paris : Colin)

Krieg-Planque, Alice. 2009. La notion de « formule » en analyse du discours. Cadre théorique et méthodologique (Besançon : P. U. de Franche-Comté)

Laforest, Marty \& Claudine Moïse. 2013. « Entre reproche et insulte, comment définir les actes de condamnation? », Fracchiolla, Béatrice, Claudine Moïse, Christina Romain \& Nathalie Auger (éds). Violences verbales. Analyses, enjeux et perspectives (Rennes : P. U. de Rennes), 85-101

Laforest, Marty \& Diane Vincent. 2004. « La qualification péjorative dans tous ses états », Langue française 144, 59-81

Lamarre, Patricia. 2018. "Montréal : terrain de lutte et/ou jeu de marelle linguistique?», Martineau, France, Annette Boudreau, Yves Frenette \& Françoise Gadet (éds). Francophonies nordaméricaines. Langues, frontières et idéologies (Québec : Presses de l'Université Laval), 89-92

Le Breton, David. 1997. Du silence (Paris : Métailié)

Le Deuff, Olivier. 2006. « Autorité et pertinence vs popularité et influence : réseaux sociaux sur Internet et mutations institutionnelles », https://archivesic.ccsd.cnrs.fr/file/index/docid/ 122603/filename/Autorite_et_institutution_vs_popularite_et_influence.pdf (consulté le 14 mars 2021)

Mercier, Arnaud. 2015. «Twitter, espace polémique, espace politique. L'exemple des tweetscampagne municipale en France (janvier-mars 2014) », Les Cahiers du numérique 4, 145-169

Moïse, Claudine, Nathalie Auger, Béatrice Fracchiolla \& Christina Schultz-Romain (éds). 2008. La violence verbale. Espaces politiques et médiatiques (Paris : L'Harmattan)

Office québécois de la langue française. 2018. Mission et rôle de l'organisme, https:// www.oqlf.gouv.qc.ca/office/mission.html (consulté le 14 mars 2021)

Paveau, Marie-Anne. 2015. «Ce qui s'écrit dans les univers numériques. Matières technolangagières et formes techno-discursives ", Itinéraires 1, https://journals.openedition.org/ itineraires/2313 (consulté le 14 mars 2021)

Paveau, Marie-Anne. 2006. Les prédiscours. Sens, mémoire et cognition (Paris : Presses Sorbonne nouvelle)

Rabatel, Alain. 2004. «L'effacement énonciatif dans les discours rapportés et ses effets pragmatiques », Langages 156, 3-17

Vincent, Diane. 2013. «L'agression verbale comme mode d'acquisition d'un capital symbolique », Fracchiolla, Béatrice, Claudine Moïse, Christina Romain \& Nathalie Auger (éds.). Violences verbales. Analyses, enjeux et perspectives (Rennes : P. U. de Rennes), 37-53 


\section{NOTES}

1. https://www12.statcan.gc.ca/census-recensement/2016/as-sa/98-200-x/2016009/98-200x2016009-fra.cfm (consulté en juillet 2020).

2. https://www150.statcan.gc.ca/n1/daily-quotidien/170817/dq170817a-fra.htm (consulté en août 2020).

3.

http://ville.montreal.qc.ca/portal/page? _pageid=6897,67887637\&_dad=portal\&_schema=PORTAL (consulté en juillet 2020).

4. http://ville.montreal.qc.ca/pls/portal/docs/PAGE/MTL_STATS_FR/MEDIA/DOCUMENTS/ 13F_LANGUES\%20DE\%20TRAVAIL_2.PDF (consulté en août 2020).

5. Organisme chargé «de définir et de conduire la politique québécoise en matière d'officialisation linguistique, de terminologie ainsi que de francisation de l'Administration et des entreprises; de veiller à ce que le français soit la langue habituelle et normale du travail, des communications, du commerce et des affaires dans l'Administration et les entreprises; d'aider à définir et à élaborer les programmes de francisation prévus par la loi et en suivre l'application ; de surveiller l'évolution de la situation linguistique au Québec et d'en faire rapport tous les cinq ans au ministre; d'assurer le respect de la Charte de la langue française, agissant d'office ou à la suite de la réception de plaintes; d'établir les programmes de recherche nécessaires à l'application de la loi et d'effectuer ou de faire effectuer les études prévues par ces programmes » (OQLF 2018 : en ligne).

6. Précisons que dans la mesure où nous avons accès au discours de Jolin-Barrette seulement par le regard des journalistes, nous nous en tenons, dans ce paragraphe, aux citations des mots du ministre.

\section{ABSTRACTS}

In November 2019, Simon Jolin-Barrette, Quebec minister responsible for the French language, revisited a controversy that had taken place two years earlier concerning the bilingual greeting bonjour/hi used in Montreal businesses, considered by some to reflect the decline of the French language in Quebec. The minister stated that he intended to ban the greeting in favor of the French ritual bonjour. In a conflicted sociolinguistic context such as Quebec's, such assertions cannot go unnoticed. The announcement of Jolin-Barrette resounded in the media, where it provoked strong reactions to such an extent that a new polemic broke out. The aim of this article is to show how the reactions provoked, especially online, contributed to the deconstruction of the minister's discourse and to its inability to impose itself as a discourse of authority. Our analysis focusses on the (techno)discursive modalities through which Jolin-Barrette's authority is diminished or denied.

En novembre 2019, Simon Jolin-Barrette, ministre québécois responsable de la langue française, revient sur une polémique qui avait eu lieu deux ans auparavant et qui portait sur la formule de salutation bilingue bonjour/hi, employée dans les commerces montréalais et considérée par certains comme signe du déclin du français au Québec. Le ministre déclare avoir l'intention d'interdire cette formule au profit du rituel français de salutation bonjour. Or, dans un contexte sociolinguistique comme celui du Québec, une telle affirmation ne peut passer inaperçue et une nouvelle polémique éclate. Notre objectif est de montrer comment les réactions suscitées - dans 
l'arène politique et les médias, mais surtout au sein de la population s'exprimant en ligne - ont contribué à la déconstruction du discours du ministre et à son incapacité à s'imposer comme discours d'autorité. Notre attention porte principalement sur les modalités (techno)discursives au moyen desquelles les participants aux échanges affaiblissent, voire nient, l'autorité du ministre.

INDEX

Mots-clés: autorité, formule discursive, médias, polémique, Québec

Keywords: authority, discursive formula, media, polemic, Quebec

\section{AUTHORS}

\section{CHIARA MOLINARI}

Università degli Studi di Milano

\section{GENEVIÈVE BERNARD BARBEAU}

Université du Québec à Trois-Rivières 